\title{
Error Bounds for Polynomial Spline Interpolation*
}

\author{
By Martin H. Schultz
}

\begin{abstract}
New upper and lower bounds for the $L^{2}$ and $L^{\infty}$ norms of derivatives of the error in polynomial spline interpolation are derived. These results improve corresponding results of Ahlberg, Nilson, and Walsh, cf. [1], and Schultz and Varga, cf. [5].
\end{abstract}

1. Introduction. In this paper, we derive new bounds for the $L^{2}$ and $L^{\infty}$ norms of derivatives of the error in polynomial spline interpolation. These bounds improve and generalize the known error bounds, cf. [1] and [5], in the following important ways: (1) these bounds can be explicitly calculated and are not merely asymptotic error bounds such as those given in [1] and [5]; (2) explicit lower bounds are given for the error for a class of functions; (3) the degree of regularity required of the function, $f$, being interpolated is extended, i.e., in [1] and [5] we demand that the $m$ th or $2 m$ th derivative of $f$ be in $L^{2}$, if we are interpolating by splines of degree $2 m-1$, while here we demand only that some $p$ th derivative of $f$, where $m \leqq p \leqq 2 m$, be in $L^{2}$; and (4) bounds are given for high-order derivatives of the interpolation errors.

2. Notations. Let $-\infty<a<b<\infty$ and for each positive integer, $m$, let $K^{m}[a, b]$ denote the collection of all real-valued functions $u(x)$ defined on $[a, b]$ such that $u \in C^{m-1}[a, b]$ and such that $D^{m-1} u$ is absolutely continuous, with $D^{m} u \in L^{2}[a, b]$, where $D u \equiv d u / d x$ denotes the derivative of $u$. For each nonnegative integer, $M$, let $\mathcal{P}_{M}(a, b)$ denote the set of all partitions, $\Delta$, of $[a, b]$ of the form

$$
\Delta: a=x_{0}<x_{1}<\cdots<x_{M}<x_{M+1}=b .
$$

Moreover, let $\mathcal{P}(a, b) \equiv \bigcup_{M=0}^{\infty} \mathcal{P}_{M}(a, b)$.

If $\Delta \in \mathcal{P}_{M}(a, b), m$ is a positive integer and $z$ is an integer such that $m-1 \leqq$ $z \leqq 2 m-2$, we define the spline space, $S(2 m-1, \Delta, z)$, to be the set of all real-valued functions $s(x) \in C^{2}[a, b]$ such that on each subinterval $\left(x_{i}, x_{i+1}\right), 0 \leqq i \leqq M, s(x)$ is a polynomial of degree $2 m-1$. We remark that our definition is identical with the definition of deficient splines of [1]. For generalizations of this concept of spline subspace, the reader is referred to [5]. In particular, it is easy to verify that all the results of this paper remain essentially unchanged if one allows the number $z$ to depend on the partition points, $x_{i}, 1 \leqq i \leqq M$, in such a way that $m-1 \leqq z\left(x_{i}\right) \leqq$ $2 m-2$ for all $1 \leqq i \leqq M$. The details are left to the reader.

Following [1] we define the interpolation mapping $\mathfrak{I}_{m}: C^{m-1}[a, b] \rightarrow S(2 m-1, \Delta, z)$ by $g_{m}(f) \equiv s$, where

$$
\begin{aligned}
D^{k} s\left(x_{i}\right) \equiv D^{k} f\left(x_{i}\right), & 0 \leqq k \leqq 2 m-2-z, \quad 1 \leqq i \leqq M, \\
& 0 \leqq k \leqq m-1, \quad i=0 \text { and } M+1 .
\end{aligned}
$$

Received February 4, 1969, revised February 2, 1970.

AMS Subject Classifications. Primary 6520, 6580.

Key Words and Phrases. Spline, interpolation, approximation.

* This research was supported in part by the National Science Foundation, GP-11326. 
We remark that the preceding interpolation mapping corresponds to the Type I interpolation of [1]. It is easy to modify the results of this paper for the cases in which the interpolation mapping corresponds to Types II, III, and IV interpolation of [1]. The details are left to the reader.

3. Basic $L^{2}$-Error Bounds. In this section, we obtain explicit upper and lower bounds for the quantities $\Lambda(m, p, z, j), 1 \leqq m, m \leqq p \leqq 2 m, m-1 \leqq z \leqq 2 m-2$, and $0 \leqq j \leqq m$, defined by

$$
\begin{array}{r}
\Lambda(m, p, z, j) \equiv \operatorname{Sup}\left\{\left\|D^{i}\left(f-\mathcal{I}_{m} f\right)\right\|_{L^{*}(a, b]} /\left\|D^{p} f\right\|_{L^{2}[a, b]}\right. \\
\left.\qquad \mid f \in K^{p}[a, b],\left\|D^{p} f\right\|_{L^{2}\{a, b]} \neq 0\right\} .
\end{array}
$$

First, we recall some basic results from [1] and [5] and introduce some additional notation.

THEOREM 3.1. The interpolation mapping given by (2.2) is well defined for all $\Delta \in \mathcal{P}(a, b), 1 \leqq m$, and $m-1 \leqq z \leqq 2 m-2$.

TheOREM 3.2 (FIRST INTEGRAL RELATION). If $f \in K^{m}[a, b], 1 \leqq m, \Delta \in \mathcal{P}(a, b)$. and $m-1 \leqq z \leqq 2 m-2$,

$$
\left\|D^{m} f\right\|_{L^{2}[a, b]}^{2}=\left\|D^{m}\left(f-g_{m} f\right)\right\|_{L^{2}(a, b]}^{2}+\left\|D^{m} g_{m} f\right\|_{L^{2}[a, b]}^{2} .
$$

THeOREM 3.3 (SECOND INTEGRAL ReLATION). If $f \in K^{2 m}[a, b], 1 \leqq m, \Delta \in \mathcal{O}(a, b)$. and $m-1 \leqq z \leqq 2 m-2$,

$$
\left\|D^{m}\left(f-\mathfrak{I}_{m} f\right)\right\|_{L \cdot[a, b]}^{2}=\int_{a}^{b}\left(f-\mathfrak{g}_{m} f\right) D^{2 m} f d x .
$$

Finally, following Kolmogorov, cf. [4, p. 146], if $t$ and $d$ are positive integers, let $\lambda_{d}(t)$ denote the $d$ th eigenvalue of the boundary value problem,

$$
\begin{aligned}
(-1)^{t} D^{2 t} y(x) & =\lambda y(x), \quad a<x<b, \\
D^{k} y(a) & =D^{k} y(b)=0, \quad t \leqq k \leqq 2 t-1,
\end{aligned}
$$

where the $\lambda_{d}$ are arranged in order of increasing magnitude and repeated according to their multiplicity. We remark that the problem (3.4)-(3.5) has a countably infinite number of eigenvalues, all of which are nonnegative and it may be shown that

$$
\lambda_{d}=(\pi /(b-a))^{2 t} d^{2 t}\left[1+O\left(d^{-1}\right)\right], \quad \text { as } t<d \rightarrow \infty .
$$

Using the bootstrapping technique of [1, p. 92], and letting

$$
\bar{\Delta} \equiv \max _{0 \leq i \leq M}\left(x_{i+1}-x_{i}\right) \quad \text { and } \quad \Delta \equiv \min _{0 \leq i \leq M}\left(x_{i+1}-x_{i}\right),
$$

for all $\Delta \in \mathcal{P}_{M}(a, b)$, we have the following generalization of Theorem 7 of [5].

THEOREM 3.4.

$$
\lambda_{d}^{-1 / 2}(m-j) \leqq \Lambda(m, m, z, j) \leqq K_{m, m, z, j}(\bar{\Delta})^{m-j},
$$

where

$$
d \equiv(M+1)(2 m-z+1)+z-j+2
$$


and

$$
\begin{array}{rlrl}
K_{m, m, z, j} & =1, & & \text { if } m-1 \leqq z \leqq 2 m-2, j=m, \\
& =(1 / \pi)^{m-i}, & & \text { if } m-1=z, 0 \leqq j \leqq m-1, \\
(3.8) & =\frac{(z+2-m) !}{\pi^{m-i}}, & & \text { if } m-1 \leqq z \leqq 2 m-2,0 \leqq j \leqq 2 m-2-z, \\
& =\frac{(z+2-m) !}{j ! \pi^{m-j}}, & \text { if } m-1 \leqq z \leqq 2 m-2,2 m-2-z \leqq j \leqq m-1,
\end{array}
$$

for all $1 \leqq m, 0 \leqq M, \Delta \in \odot_{M}(a, b), m-1 \leqq z \leqq 2 m-2$, and $0 \leqq j \leqq m$.

Proof. First, we prove the right-hand inequality of (3.6). If $m-1 \leqq z \leqq 2 m-2$ and $j=m$, the result follows directly from Theorem 3.2.

Otherwise, $D^{i}\left(f-g_{m} f\right)\left(x_{i}\right)=0,1 \leqq i \leqq M, 0 \leqq j \leqq 2 m-2-z$, and by the Rayleigh-Ritz inequality, cf. [3, p. 184],

$$
\int_{x_{i}}^{x_{i+1}}\left(D^{i}\left(f-g_{m} f\right)(x)\right)^{2} d x \leqq\left(\frac{\bar{\Delta}}{\pi}\right)^{2} \int_{x_{i}}^{x_{i+1}}\left(D^{i+1}\left(f-g_{m} f\right)(x)\right)^{2} d x,
$$

$0 \leqq j \leqq 2 m-2-z$. Summing both sides of (3.9) with respect to $i$ from 0 to $M$, we obtain

$$
\left\|D^{j}\left(f-\mathscr{I}_{m} f\right)\right\|_{L^{2}\{a, b\}} \leqq \frac{\bar{\Delta}}{\pi}\left\|D^{j+1}\left(f-\mathscr{I}_{m} f\right)\right\|_{L^{2}\{a, b\}},
$$

$0 \leqq j \leqq 2 m-2-z$. Using (3.10) repeatedly we obtain

$$
\left\|D^{i}\left(f-\mathfrak{g}_{m} f\right)\right\|_{L^{2}\{a, b]} \leqq\left(\frac{\bar{\Delta}}{\pi}\right)^{2 m-1-z-i}\left\|D^{2 m-1-z}\left(f-\mathfrak{I}_{m} f\right)\right\|_{L^{2}[a, b]} .
$$

Hence, if $2 m-1-z=m$, i.e., $z=m-1$, then

$$
\left\|D^{i}\left(f-\mathfrak{I}_{m} f\right)\right\|_{L^{2}\{a, b]} \leqq\left(\frac{1}{\pi}\right)^{m-i}(\bar{\Delta})^{m-j}\left\|D^{m} \int\right\|_{L^{2}\{a, b\}}
$$

which is the required result for this special case.

Otherwise, since $m \leqq z$, applying Rolle's Theorem to $D^{2 m-2-z}\left(f-g_{m} f\right) \in$ $C^{x-m+1}[a, b]$, which vanishes at every mesh point, we have that for each $0 \leqq j \leqq$ $z-m+1$, there exist points $\left\{\xi_{l}^{(i)}\right\}_{l=0}^{M+1-i}$ in $[a, b]$ such that

$$
\begin{aligned}
& D^{2 m-2-z+i}\left(f-g_{m} f\right)\left(\xi_{l}^{(j)}\right)=0, \quad 0 \leqq j \leqq m-1-(2 m-2-z), \\
&=z-m+1, \quad 0 \leqq l \leqq M+1-j, \\
& a=\xi_{0}^{(j)}<\xi_{1}^{(j)}<\cdots<\xi_{. M+1-j}^{(j)}=b, \quad 0 \leqq j \leqq z-m+1, \\
& \xi_{l}^{(j)} \leqq \xi_{l}^{(j+1)}<\xi_{l+1}^{(j)}, \quad \text { for all } 0 \leqq l \leqq M+1-j, 0 \leqq j \leqq z-m+1
\end{aligned}
$$

and

$$
\left|\xi_{l+1}^{(i)}-\xi_{l}^{(i)}\right| \leqq(j+1) \bar{\Delta}, \quad 0 \leqq l \leqq M-j, 0 \leqq j \leqq z-m+1
$$

i.e., choose $\xi_{l}^{(0)}=x_{l}, 0 \leqq l \leqq M+1$. 
Thus, applying the Rayleigh-Ritz inequality, we have

$$
\begin{aligned}
\int_{\xi_{l}(i)}^{\xi_{l+1}(i)}\left(D^{2 m-2-s+j}\left(f-g_{m} f\right)(x)\right)^{2} d x \\
\leqq\left[\frac{(j+1) \bar{\Delta}}{\pi}\right]^{2} \int_{\xi_{1}(j)}^{\xi_{(i+1)}(f)}\left(D^{2 m-2-s+(j+1)}\left(f-g_{m} f\right)\right)^{2} d x
\end{aligned}
$$

for all $0 \leqq l \leqq M-j, 0 \leqq j \leqq z-m+1$. Summing (3.17) with respect to $l$ from 0 to $M-j$, we have

$$
\cdot\left\|D^{2 m-2-\varepsilon+i}\left(f-g_{m} f\right)\right\|_{L^{*}[a, b]} \leqq \frac{(j+1) \bar{\Delta}}{\pi}\left\|D^{2 m-2-s+(j+1)}\left(f-g_{m} f\right)\right\|_{L \cdot[a, b]},
$$

$0 \leqq j \leqq z-m+1$. Using (3.18) repeatedly along with (3.2) we have

$$
\begin{aligned}
\left\|D^{2 m-1-z}\left(f-g_{m} f\right)\right\|_{L^{*}[a, b]} & \leqq \frac{(z+2-m) !}{\pi^{z-m+1}}(\bar{\Delta})^{z-m+1}\left\|D^{m}\left(f-g_{m} f\right)\right\|_{L \cdot\{a, b]} \\
& \leqq \frac{(z+2-m) !}{\pi^{s-m+1}}(\bar{\Delta})^{s-m+1}\left\|D^{m} f\right\|_{L^{*}[a, b]} .
\end{aligned}
$$

Combining (3.11) with (3.19), we have that

$$
\left\|D^{i}\left(f-g_{m} f\right)\right\|_{L^{\cdot}[a, b]} \leqq \frac{(z+2-m) !}{\pi^{m-i}}(\bar{\Delta})^{m-i}\left\|D^{m} f\right\|_{L \cdot(a, b)},
$$

if $0 \leqq j \leqq 2 m-2-z$. Otherwise, it follows from (3.18) that

$$
\left\|D^{i}\left(f-\mathcal{I}_{m} f\right)\right\|_{L \cdot[a, b]} \leqq \frac{(z+2-m) !}{j ! \pi^{m-i}}\left\|D^{m} f\right\|_{L^{\cdot}[a, b]} .
$$

Finally, we prove the left-hand inequality of (3.6). This inequality follows directly from a fundamental result of Kolmogorov, cf. [4, p. 146], which states that

$$
\lambda_{t+1}^{-1 / 2}(m-j) \leqq \Lambda(m, m, z, j),
$$

where $t \equiv$ dimension $D^{i}(S(2 m-1, \Delta, z))$, for all $1 \leqq m, 0 \leqq M, \Delta \in \mathcal{P}_{M}(a, b)$, $m-1 \leqq z \leqq 2 m-2$, and $0 \leqq j \leqq m$. But the space $D^{i}(S(2 m-1, \Delta, z))$ bas dimension $t \equiv(2 m-j)(M+1)-(z+1-j) M=(M+1)(2 m-z+1)+z-j+1$. Q.E.D.

We remark that in this case it is easy to verify that there exists a positive constant, $K$, such that

$$
\begin{aligned}
\lambda_{d}^{-1 / 2} & \geqq\left(\frac{b-a}{\pi}\right)^{m-j} \frac{1}{(M+1)^{m-i}} \frac{1}{s^{m-i}} \frac{1}{1+K s^{-1}(M+1)^{-1}} \\
& \geqq \frac{1}{\pi^{m-i}} \frac{1}{s^{m-i}} \frac{1}{1+K s^{-1}(M+1)^{-1}}(\Delta)^{m-i},
\end{aligned}
$$

where $s \equiv(2 m-z+1+(z-j+2) /(M+1))$, and thus that splines are "quasioptimal".

The next result generalizes Theorem 9 of [5].

THEOREM 3.5 .

$$
\lambda_{d}^{-1 / 2}(2 m-j) \leqq \Delta(m, 2 m, z, j) \leqq K_{m, 2 m, . . ;}(\Delta)^{2 m-i}
$$


where

$$
d \equiv(M+1)(2 m-z+1)+z-j+2
$$

and

$$
\begin{array}{r}
K_{m, 2 m, s, j} \equiv\left(K_{m, m, s, i}\right)\left(K_{m, m, z, 0}\right), \text { for all } 1 \leqq m, 0 \leqq M, \Delta \in \mathcal{P}_{M}(a, b), \\
m-1 \leqq z \leqq 2 m-2, \text { and } 0 \leqq j \leqq m .
\end{array}
$$

Proof. Applying the Cauchy-Schwarz inequality to the Second Integral Relation yields the inequality

$$
\left\|D^{m}\left(f-g_{m} f\right)\right\|_{L \cdot\{a, b]}^{2} \leqq\left\|D^{2 m} f\right\|_{L^{\prime}[a, b]}\left\|f-\mathscr{g}_{m} f\right\|_{L^{\prime}[a, b]} .
$$

Applying the proof of Theorem 3.4, we have

$$
\left\|D^{i}\left(f-g_{m} f\right)\right\|_{L^{\prime}\{a, b]} \leqq K_{m, m, z, j}\left\|D^{m}\left(f-g_{m} f\right)\right\|_{L^{\prime}\{a, b\}}(\bar{\Delta})^{m-i} .
$$

Using (3.27) for the special case of $j=0$ in (3.26) yields

$$
\left\|D^{m}\left(f-g_{m} f\right)\right\|_{L \cdot[a, b]} \leqq\left\|D^{2 m} f\right\|_{L^{2}[a, b]} K_{m, m, 8,0}(\bar{\Delta})^{m} .
$$

Using (3.28) to bound the right-hand side of (3.27) gives us the right-hand inequality of (3.23). The left-hand inequality of (3.23) follows as in Theorem 3.4. Q.E.D.

We now recall a fundamental inequality of E. Schmidt which will be used several times in the remainder of this paper.

LEMMA 3.1. If $p_{N}(x)$ is a polynomial of degree $N$,

$$
\left\|D p_{N}\right\|_{L \cdot\{a, b\}} \leqq \frac{E_{N}}{b-a}\left\|p_{N}\right\|_{L^{2}\{a, b]},
$$

where $E_{N} \equiv(N+1)^{2} \sqrt{ } 2$.

Proof. Cf. [2]. Q E.D.

THEOREM 3.6.

$$
\lambda_{d}^{-1 / 2}(p-j) \leqq \Lambda(m, p, z, j) \leqq K_{m, p, s, i}(\bar{\Delta})^{p-j},
$$

where

$$
d \equiv(M+1)(2 m-z+1)+z-j+2
$$

and

$$
K_{m, p, \&, i} \equiv\left\{K_{p, p, 2 m-1, i}+K_{m, 2 m, 8, i} \cdot 2^{(1 / 2)(2 m-p)}\left[\frac{p !}{(2 p-2 m) !}\right]^{2}(\bar{\Delta} / \Delta)^{2 m-p}\right\}
$$

for all $1 \leqq m, 0 \leqq M, \Delta \in \mathcal{P}_{M}(a, b), m<p<2 m, 4 m-2 p-1 \leqq z \leqq 2 m-2$, and $0 \leqq j \leqq m$.

Proof. Consider $S(2 p-1, \Delta, 2 m-1) \subset K^{2 m}[a, b]$. This space is well defined since $2 p-2 \geqq 2(m+1)-2=2 m$. Moreover, if $\mathscr{J}_{m}$ denotes the interpolation mapping of $C^{m-1}[a, b]$ into $S(2 m-1, \Delta, z)$ and $\mathscr{I}_{p}$ denotes the interpolation mapping of $C^{p-1}[a, b]$ into $S(2 p-1, \Delta, 2 m-1)$, then $\mathscr{g}_{m}\left(g_{p} f\right)=g_{m} f$ for all $f \in C^{p-1}[a, b]$. In fact, $D^{k} \mathcal{G}_{p} f$ interpolates $D^{k} f$ at $x_{i}, 1 \leqq i \leqq M$, for all $0 \leqq k \leqq 2 p-(2 m-1)-$ $2=2 p-2 m-1$, while $D^{k} \mathscr{g}_{m} f$ interpolates $D^{k} f$ at $x_{i}, 1 \leqq i \leqq M$, for all $0 \leqq k \leqq$ $2 m-z-2 \leqq 2 m-(4 m-2 p-1)-2=2 p-2 m-1$. 
Thus,

$$
\begin{aligned}
\left\|D^{j}\left(f-g_{m} f\right)\right\|_{L^{2}(a, b)} \leqq & \left\|D^{j}\left(f-g_{p} f\right)\right\|_{L^{\prime}(a, b)} \\
& +\left\|D^{i}\left(g_{p} f-g_{m}\left(g_{p} f\right)\right)\right\|_{L^{\prime}(a, b)}, \quad 0 \leqq j \leqq m .
\end{aligned}
$$

By Theorem 3.4,

$$
\left\|D^{i}\left(f-g_{p} f\right)\right\|_{L^{2}[a, b]} \leqq K_{p, D, 2 m-1, j}(\bar{\Delta})^{p-i}\left\|D^{p} f\right\|_{L=1 a, b]},
$$

and by Theorem 3.5

$$
\left\|D^{i}\left(g_{p} f-\mathscr{G}_{m}\left(\mathscr{g}_{p} f\right)\right)\right\|_{L \cdot[a, b]} \leqq K_{m, 2 m, 2, j}(\bar{\Delta})^{2 m-i}\left\|D^{2 m} g_{p} f\right\|_{L^{2}[a, b]}
$$

But by Schmidt's inequality and the First Integral Relation, since $\mathscr{g}_{p} f$ is a piecewise polynomial of degree $2 p-1$ with $p>m$, we have

$$
\begin{aligned}
\left\|D^{2 m} \mathscr{g}_{p} f\right\|_{L^{2}(a, b)} & \leqq \frac{\left(\prod_{i=1}^{2 m-p} E_{2 p-2 m-1+i}\right)\left\|D^{p} f\right\|_{L^{2}(a, b)}}{(\Delta)^{2 m-p}} \\
& \leqq 2^{(2 m-p) / 2}\left[\frac{p !}{(2 p+2 m) !}\right]^{2} \frac{\left\|D^{p} f\right\|_{L^{2}[a, b]}}{(\Delta)^{2 m-p}} .
\end{aligned}
$$

The required result now follows from (3.33), (3.34), (3.35), and (3.36). Q.E.D.

4. $L^{2}$-Error Bounds for Higher Order Derivatives. In this section we give explicit upper bounds for the quantities $\Lambda(m, p, z, j)$ in the special cases of $m<p \leqq 2 m$ and $m<j \leqq p$. Since $g_{m} f$ is not necessarily in $K^{i}[a, b]$ if $z+1<j \leqq p$, it is necessary to modify the definition of $\Lambda(m, p, z, j)$ given in (3.1). The new definition is given by

$$
\Lambda(m, p, z, j) \equiv \operatorname{Sup}\left\{\left(\sum_{i=0}^{M}\left\|D^{j}\left(f-\mathcal{I}_{m} f\right)\right\|_{L^{2}\left[x_{i}, x_{i+1}\right\}}^{2}\right)^{1 / 2} /\left\|D^{p} f\right\|_{L^{2}[a, b]}\right.
$$

$$
\left.\mid f \in K^{p}[a, b],\left\|D^{p} f\right\|_{L^{2}(a, b]} \neq 0\right\} \text {. }
$$

The main result of this section is

THEOREM 4.1.

$$
\Lambda(m, p, z, j) \leqq K_{m, p, z, j}(\bar{\Delta})^{p-i},
$$

where

$$
K_{m, p, z, j} \equiv\left[K_{p, p, p, j}+\left(K_{m, p, z, m}+K_{p, p, p, m}\right) 2^{(i-m) / 2}\left[\frac{(2 p+m) !}{(2 p-j) !}\right]^{2}\left(\frac{\bar{\Delta}}{\Delta}\right)^{i-m}\right],
$$

for all $1 \leqq m, 0 \leqq M, \Delta \in \mathcal{P}_{M}(a, b), m<p \leqq 2 m, 4 m-2 p-1 \leqq z \leqq 2 m-2$. and $m<j \leqq p$.

Proof. By Theorem 3.6,

$$
\left\|D^{m}\left(f-\mathscr{g}_{m} f\right)\right\|_{L^{2}|a, b|} \leqq K_{m, p, z, m}(\bar{\Delta})^{p-m},
$$

and by Theorem 3.4 ,

$$
\left\|D^{k}\left(f-\mathscr{G}_{p} f\right)\right\|_{L^{2}\{a, b\}} \leqq K_{p, p, p, k}(\bar{\Delta})^{p-k}, \quad 0 \leqq k \leqq p .
$$


Combining (4.4) and (4.5), we obtain

$$
\left\|D^{m}\left(g_{m} f-g_{p} f\right)\right\|_{L^{2}\{a, b]} \leqq\left(K_{m, p, 2, m}+K_{p, p, p, m}\right)(\bar{\Delta})^{p-m} .
$$

Using the Schmidt inequality in (4.6), we obtain

$$
\begin{aligned}
& \left\|D^{j}\left(\mathfrak{g}_{m} f-\mathfrak{g}_{p} f\right)\right\|_{L^{2}|a, b|} \leqq \frac{\left(\prod_{i=1}^{i-m} E_{(2 p-1)-i+i}\right)}{(\underline{\Delta})^{i-m}}\left\|D^{m}\left(\mathfrak{I}_{m} f-\mathfrak{g}_{p} f\right)\right\|_{\left.L^{x} \mid a, b\right]} \\
& \leqq\left(K_{m, p, z, m}+K_{p, p, p, m}\right)\left(\prod_{i=1}^{i-m} E_{2 p-1-i+i}\right)(\bar{\Delta})^{\nu-i}(\bar{\Delta} / \Delta)^{i-m} .
\end{aligned}
$$

The required result follows from (4.5), (4.7), and

$$
\left\|D^{i}\left(f-g_{m} f\right)\right\|_{L^{2}[a, b]} \leqq\left\|D^{i}\left(f-g_{p} f\right)\right\|_{L^{*}(a, b]}+\left\|D^{i}\left(g_{p} f-g_{m} f\right)\right\|_{L^{*}[a, b]} .
$$

Q.E.D.

We remark that in those cases in which $g_{m} f \in K^{i}[a, b]$, lower bounds of the form introduced in Section 3 can be given for $\Lambda(m, p, z, j)$.

5. $L^{\infty}$-Error Bounds. In this section, we give explicit upper bounds for the quantities $\Lambda^{\infty}(m, p, z, j), 1 \leqq m, m \leqq p \leqq 2 m, m-1 \leqq z \leqq 2 m-2$, and $0 \leqq j \leqq p$, defined by

$$
\begin{array}{r}
\Lambda^{\infty}(m, p, z, j) \equiv \operatorname{Sup}\left\{\max _{0 \leqq i \leq M}\left(\left\|D^{i}\left(f-g_{m} f\right)\right\|_{L^{\infty}\left[x_{i}, x_{i+1}\right]}\right) /\left\|D^{p} f\right\|_{\left.L^{2} \mid a, b\right]}\right. \\
\\
\left.\quad f \in K^{p}[a, b],\left\|D^{p} f\right\|_{\left.L^{2} \mid a, b\right\}} \neq 0\right\} .
\end{array}
$$

We obtain the following results as corollaries of the results of Section 3 and Section 4 . As an improvement of Theorem 6 of [5], we have

THEOREM 5.1.

$$
\Lambda^{\infty}(m, m, z, j) \leqq K_{m, m, z, j}^{\infty}(\bar{\Delta})^{m-j-1 / 2},
$$

where

$$
\begin{aligned}
K_{m, m, z, j}^{\infty} \equiv K_{m, m, z, j+1}, \quad \text { if } m-1=z, 0 \leqq j \leqq m-1, \\
\equiv K_{m, m, z, j+1}, \quad \text { if } m-1<z \leqq 2 m-2,0 \leqq j \leqq 2 m-2-z, \\
\equiv(j-2 m+3+z)^{1 / 2} K_{m, m, z, j+1}, \quad \text { if } m-1<z \leqq 2 m-2, \\
\quad 2 m-2-z<j \leqq m-1,
\end{aligned}
$$

for all $1 \leqq m, 0 \leqq M, \Delta \in \mathcal{P}_{M}(a, b), m-1 \leqq z \leqq 2 m-2$, and $0 \leqq j \leqq m-1$

Proof. We give the proof in the special case of $m-1=z, 0 \leqq j \leqq m-1$, as the proof in the other cases is analogous. Given any $x \in[a, b]$, there exists a point $y \in[a, b]$ such that $D^{i}\left(f-g_{m} f\right)(y)=0$ and $|x-y| \leqq \bar{\Delta}$. Hence, $D^{i}\left(f-\mathfrak{g}_{m} f\right)(x)=$ $\int_{y}^{x} D^{i+1}\left(f-g_{m} f\right)(t) d t$ and

$$
\left\|D^{i}\left(f-\mathscr{g}_{m} f\right)\right\|_{L^{\infty}[a, b]} \leqq(\bar{\Delta})^{1 / 2}\left\|D^{i+1}\left(f-\mathscr{I}_{m} f\right)\right\|_{L^{2}[a, b]} .
$$

The result now follows from applying Theorem 3.4 to the right-hand side of the preceding inequality. Q.E.D. 
As in Theorem 5.1, we have as an improvement of Theorem 8 of [5]. THEOREM 5.2.

$$
\Lambda^{\infty}(m, 2 m, z, j) \leqq K_{m, 2 m, z, j}^{\infty}(\bar{\Delta})^{2 m-j-1 / 2},
$$

where

$$
\begin{aligned}
K_{m, 2 m, z, j+1}^{\infty} & \equiv K_{m, 2 m, z, j+1}, \quad \text { if } m-1=z, 0<j \leqq m-1, \\
& \equiv K_{m, 2 m, z, j+1}, \quad \text { if } m-1<z \leqq 2 m-2,0 \leqq j \leqq 2 m-2-z, \\
& \equiv(j-2 m+3+z)^{1 / 2} K_{m, 2 m, z, j+1}, \quad \text { if } m-1<z \leqq 2 m-2, \\
& 2 m-2-z<j \leqq m-1,
\end{aligned}
$$

for all $1<m, 0 \leqq M, \Delta \in \mathcal{O}_{M}(a, b), m-1 \leqq z \leqq 2 m-2$, and $0 \leqq j \leqq m-1$.

As in Theorem 3.6, we have

THEOREM 5.3 .

$$
\Lambda^{\infty}(m, p, z, j) \leqq K_{m, D, s, j}^{\infty}(\bar{\Delta})^{p-i-1 / 2},
$$

where

$$
K_{m, p, s, i}^{\infty} \equiv\left\{K_{p, p, 2 m-1,1}^{\infty}+K_{m, 2 m, s, i}^{\infty} \cdot 2^{(2 m-p) / 2}\left[\frac{p !}{(2 p-2 m) !}\right]^{2}\left(\frac{\bar{\Delta}}{\Delta}\right)^{2 m-p}\right\},
$$

for all $1 \leqq m, 0 \leqq M, \Delta \in \mathcal{P}_{M}(a, b), m<p<2 m, 4 m-2 p-1 \leqq z \leqq 2 m-2$, and $0 \leqq j \leqq m-1$.

Finally, to give a result analogous to Theorem 4.1 , we need an inequality due to A. A. Markov.

Lemma 5.1. If $p_{N}(x)$ is a polynomial of degree $N$, then

$$
\left\|D P_{N}\right\|_{L^{\infty}[a, b]} \leqq \frac{M_{N}}{b-a}\left\|p_{N}\right\|_{L^{\infty}[a, b]},
$$

where $M_{N} \equiv 2 N^{2}$.

Proof. Cf. [6]. Q.E.D.

As an extension of Theorem 10 of [5], we prove

THEOREM 5.4.

$$
\Lambda^{\infty}(m, p, z, j) \leqq K_{m, p, s, i}^{\infty}(\bar{\Delta})^{p-j-1 / 2},
$$

where

$$
K_{m, p, s, i}^{\infty} \equiv\left\{K_{p, p, p, i}^{\infty}+\left(K_{m, p, s, i}^{\infty}+K_{p, p, p, j}^{\infty}\right) 2^{i-m+1}\left(\frac{(2 p-m) !}{(2 p-j-1) !}\right)^{2}\left(\frac{\bar{\Delta}}{\Delta}\right)^{i-m+1}\right\}
$$

for all $1 \leqq m, 0 \leqq M, \Delta \in \mathcal{P}_{M}(a, b), m<p \leqq 2 m, 4 m-2 p-1 \leqq z \leqq 2 m-2$ and $m \leqq j \leqq p-1$.

Proof. From Theorem 5.1, we have that

$$
\left\|D^{k}\left(f-g_{p} f\right)\right\|_{L^{\infty}[a, b]} \leqq K_{p, p, D, k}^{\infty}(\bar{\Delta})^{p-k-1 / 2}\left\|D^{p} f\right\|_{L \cdot\{a, b]}, \quad 0 \leqq k \leqq p-1,
$$
and from Theorem 5.3

$$
\left\|D^{m-1}\left(f-g_{m} f\right)\right\|_{L^{\infty}[a, b]} \leqq K_{m, p, s, m-1}^{\infty}(\bar{\Delta})^{p-m+1 / 2}\left\|D^{p} f\right\|_{L \cdot[a, b]} .
$$


Combining (5.11) and (5.12), we have

$$
\left\|D^{m-1}\left(g_{m} f-g_{D} f\right)\right\|_{L^{\infty}[a, b]} \leqq\left(K_{m, D, Z, m-1}^{\infty}+K_{p, D, D, K}^{\infty}\right)(\bar{\Delta})^{p-m+1 / 2}\left\|D^{p} f\right\|_{L \cdot[a, b]} .
$$

But,

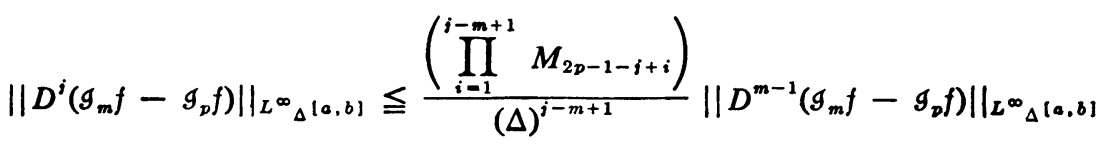

$$
\begin{aligned}
& \leqq 2^{i-m+1}\left(\frac{(2 p-m) !}{(2 p-j-1) !}\right)^{2} \frac{1}{(\Delta)^{j-m+1}} \\
& \cdot\left\|D^{m-1}\left(g_{m} f-g_{p} f\right)\right\|_{L \omega_{\Delta}(a, b]},
\end{aligned}
$$

where

$$
\|\cdot\|_{L^{\infty} \Delta[0, b]} \equiv \max _{0 \leq i \leq m}\|\cdot\|_{L^{\infty}\left[x_{\left.i, x_{i+1}\right]}\right.} .
$$

The required result follows directly from (5.11), (5.13), (5.14), and the observation that

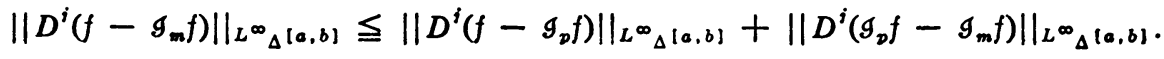

Q.E.D.

Computer Science Department

Yale University

New Haven, Connecticut 06509

1. J. H. Ahlberg, E. N. Nilson \& J. L. Walsh, The Theory of Splines and Their Applications, Academic Press, New York, 1967. MR 39 \#684.

2. R. Bellman, "A note on an inequality of E. Schmidt," Bull. Amer. Math. Soc., v. 50, 1944, pp. 734-736. MR 6, 61.

3. G. H. HaRdy, J. E. Littlewood \& G. Pólya, Inequalities, 2nd ed., Cambridge Univ. Press, New York, 1952. MR 13, 727.

4. G. G. LORENTZ, Approximation of Functions, Holt, Rinehart, and Winston, New York, 1966. MR 35 \#4642.

5. M. H. Schultz \& R. S. VARGA, "L-splines," Numer. Math., v. 10, 1967, pp. 345-369. MR 37 \#665.

6. J. ToDd (Editor), A Survey of Numerical Analysis, McGraw-Hill, New York, 1962. MR 24 \#B1271. 